ARTICLE

DOI: $10.1038 / s 41467-017-01012-6$

\title{
Evidence of indirect gap in monolayer $\mathrm{WSe}_{2}$
}

\author{
Wei-Ting Hsu', Li-Syuan Lu', Dean Wang ${ }^{1}$, Jing-Kai Huang ${ }^{2}$, Ming-Yang Li ${ }^{3}$, Tay-Rong Chang ${ }^{4}$, Yi-Chia Chou (D) 1 , \\ Zhen-Yu Juang ${ }^{1}$, Horng-Tay Jeng ${ }^{5}$, Lain-Jong Li id ${ }^{2} \&$ Wen-Hao Chang (iD) ${ }^{1}$
}

Monolayer transition metal dichalcogenides, such as $\mathrm{MoS}_{2}$ and $\mathrm{WSe}_{2}$, have been known as direct gap semiconductors and emerged as new optically active materials for novel device applications. Here we reexamine their direct gap properties by investigating the strain effects on the photoluminescence of monolayer $\mathrm{MoS}_{2}$ and $\mathrm{WSe}_{2}$. Instead of applying stress, we investigate the strain effects by imaging the direct exciton populations in monolayer $W \mathrm{Se}_{2}-\mathrm{MoS}_{2}$ and $\mathrm{MoSe}_{2}-\mathrm{WSe}_{2}$ lateral heterojunctions with inherent strain inhomogeneity. We find that unstrained monolayer $\mathrm{WSe}_{2}$ is actually an indirect gap material, as manifested in the observed photoluminescence intensity-energy correlation, from which the difference between the direct and indirect optical gaps can be extracted by analyzing the exciton thermal populations. Our findings combined with the estimated exciton binding energy further indicate that monolayer $\mathrm{WSe}_{2}$ exhibits an indirect quasiparticle gap, which has to be reconsidered in further studies for its fundamental properties and device applications.

\footnotetext{
${ }^{1}$ Department of Electrophysics, National Chiao Tung University, Hsinchu 30010, Taiwan. ${ }^{2}$ Physical Sciences and Engineering, King Abdullah University of Science and Technology, Thuwal 23955-6900, Saudi Arabia. ${ }^{3}$ Research Center for Applied Sciences, Academia Sinica, Taipei 10617, Taiwan. ${ }^{4}$ Department of Physics, National Cheng Kung University, Tainan 70101, Taiwan. ${ }^{5}$ Department of Physics, National Tsing Hua University, Hsinchu 30013, Taiwan.

Correspondence and requests for materials should be addressed to W.-H.C. (email: whchang@mail.nctu.edu.tw)
} 
T wo-dimensional (2D) transition metal dichalcogenides (TMDs) in the family of $\mathrm{MX}_{2}$ (M:Mo, W; X:S, Se) have been discovered as a new class of semiconductors for atomically thin electronics and optoelectronics ${ }^{1-6}$. Of particular interest are monolayer $\mathrm{MX}_{2}$, which have been identified as direct gap semiconductors and emerged as new optically active materials for developing novel 2D light emitters and absorber ${ }^{5,6}$. Experimental evidence of direct gap in monolayer $\mathrm{MX}_{2}$ was obtained from the observation of strongly enhanced photoluminescence (PL) caused by the indirect to direct gap transition when $\mathrm{MX}_{2}$ were thinned to one monolayer ${ }^{7}$, 8. Although most theoretical calculations have also shown a direct gap at $\mathrm{K}$-valley in all monolayer $\mathrm{MX}_{2}{ }^{9-12}$, subtle differences have been found in some calculations for monolayer $\mathrm{WSe}_{2}{ }^{13}$, where the conduction band $(\mathrm{CB})$ minimum lies at Q-valley (about midway between $\mathrm{K}$ and $\Gamma$ ). Indeed, recent scanning tunneling spectroscopy (STS) suggested that the Q-valley is about $80 \mathrm{meV}$ below the K-valley in the $\mathrm{CB}$ of monolayer $\mathrm{WSe}_{2}{ }^{14}$, indicative of an indirect quasiparticle gap. However, due to the unusually strong Coulomb interactions and the lack of knowledge on the binding energy of indirect excitons, it remains unclear whether the optical gap (i.e., quasiparticle gap minus exciton binding energy) is also indirect. While efficient PL is invariably observed in monolayer $\mathrm{WSe}_{2}$, the indirect quasiparticle gap observed by STS suggested that the direct/indirect nature of optical gap in monolayer $\mathrm{WSe}_{2}$ has not been settled thus far.

Strain is an effective perturbation that can modulate not only the direct gap of $\mathrm{MX}_{2}$, but also the indirect gap with a different a

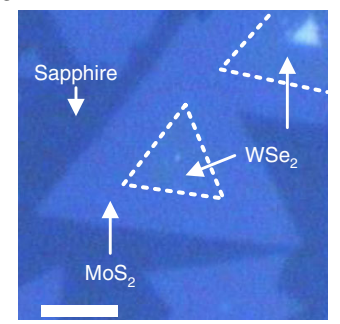

b

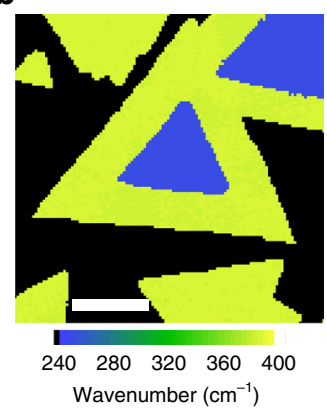

d

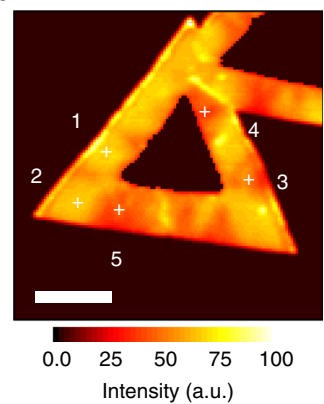

e

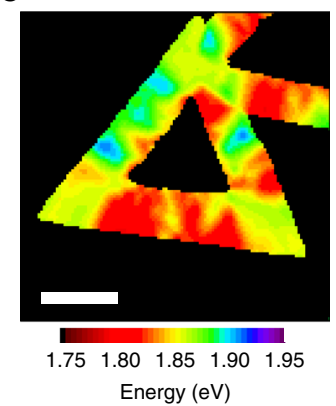

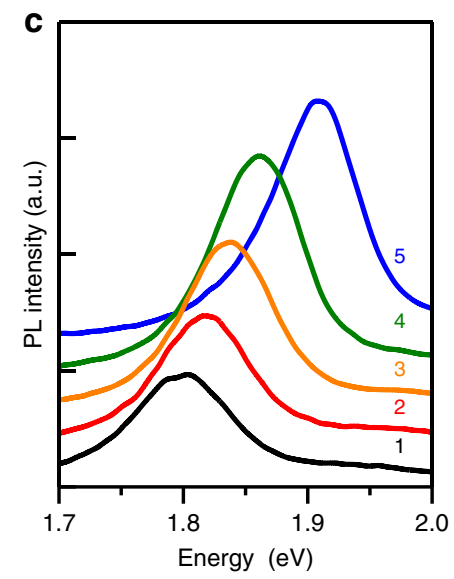

f

Energy shift $\delta E_{\mathrm{X}, \mathrm{D}}(\mathrm{meV})$

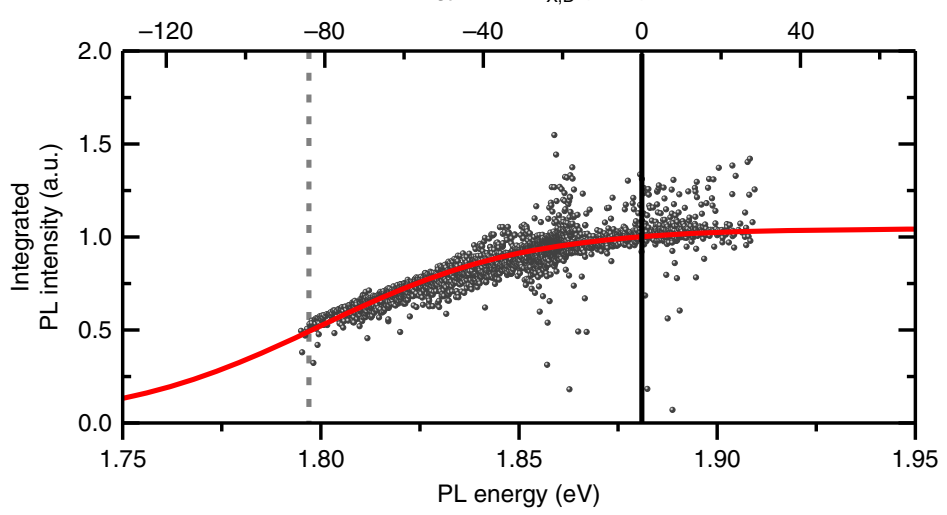

g

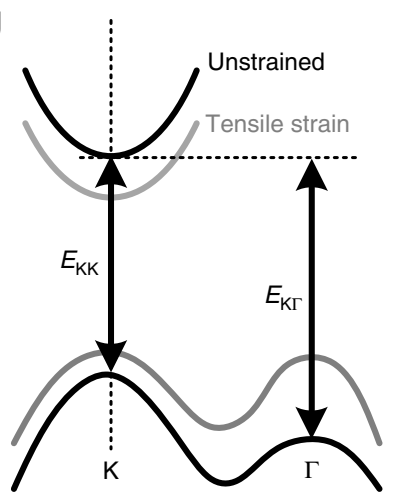

h

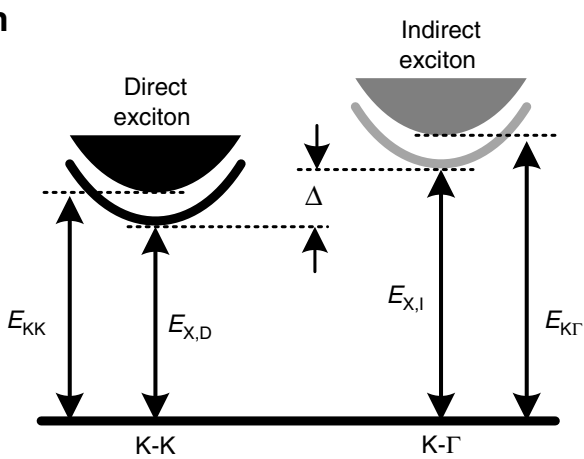

Fig. $1 \mathrm{PL}$ inhomogeneity in strained $\mathrm{WSe}_{2}-\mathrm{MoS}_{2}$ lateral HJs. a, b Optical image (a) and the corresponding contour color map of the $\mathrm{E}_{2 \mathrm{~g}}$ Raman peak position (b) of a monolayer $\mathrm{WSe}_{2}-\mathrm{MoS}_{2}$ lateral $\mathrm{HJ}$ flake. $\mathbf{c}$ Five selected $\mathrm{PL}$ spectra from different positions in the $\mathrm{MoS}_{2}$ region. $\mathbf{d}$, e Contour color maps of the PL intensity (d) and peak energy (e) of the $\mathrm{MoS}_{2}$ region. $\mathbf{f}$ Integrated PL intensity as a function of peak energy obtained from all PL spectra (black dots). Solid line is the model fitting curve. The top axis is the PL peak energy shift with respect to the unstrained $\mathrm{MoS}_{2}$ (vertical line). $\mathbf{g}$ Schematics for the band structures of monolayer $\mathrm{MoS}_{2}$ without (black) and with (gray) tensile strain. $\mathbf{h}$ Schematics for the direct $(\mathrm{K}-\mathrm{K}$ ) and indirect $(\mathrm{K}-\Gamma)$ excitonic states with energies of $E_{X, D}$ and $E_{X, 1}$, respectively 
a

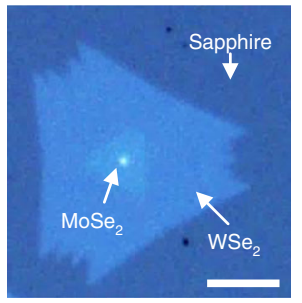

b

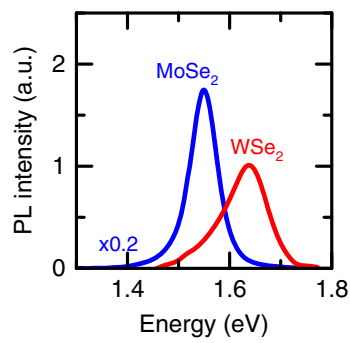

c

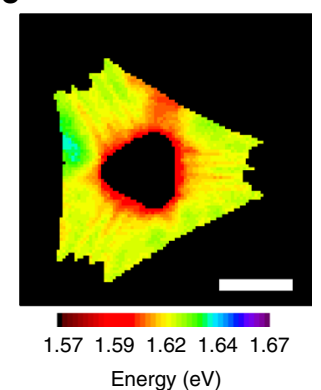

d

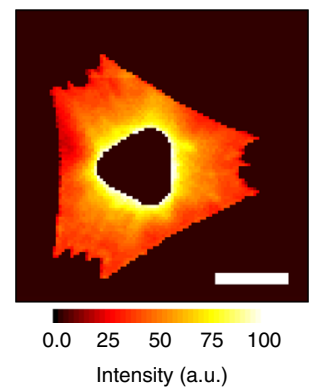

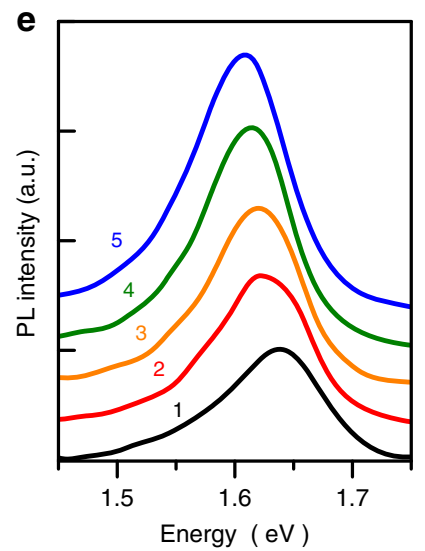

g

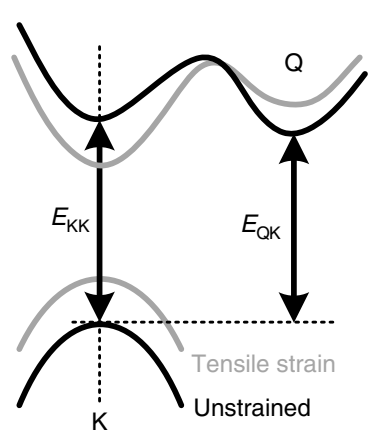

f

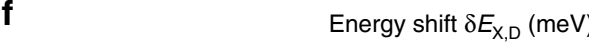

h

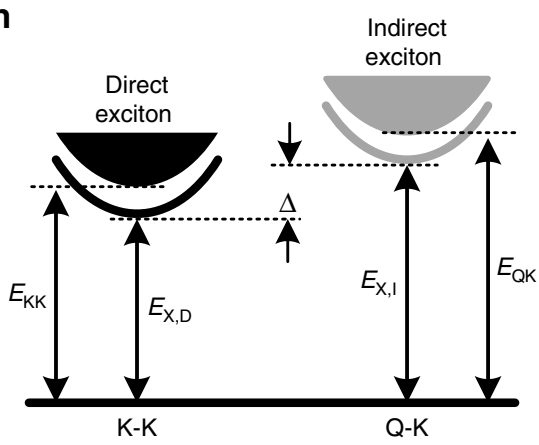

Fig. $2 \mathrm{PL}$ inhomogeneity in strained $\mathrm{MoSe}_{2}-\mathrm{WSe}_{2}$ lateral $\mathrm{HJs}$. a Optical image of a monolayer $\mathrm{MoSe}_{2}-\mathrm{WSe}_{2} \mathrm{HJ}$. b The PL spectra of the inner MoSe $e_{2}$ and the outer $\mathrm{WSe}_{2}$. c, $\mathbf{d}$ Contour color maps of the PL peak energy (c) and intensity (d) of outer WSe $\mathrm{S}_{2}$. e Five selected PL spectra from the outer WSe (f) Integrated PL intensity as a function of peak energy (black dots). Solid line is the model fitting curve. The top axis is the PL peak energy shift with respect to the unstrained $\mathrm{WSe}_{2} \cdot \mathbf{g}$ Schematics for the band structures of monolayer $\mathrm{WSe}_{2}$ without (black) and with (gray) tensile strain. $\mathbf{h} \mathrm{Schematics}$ for the direct $(\mathrm{K}-\mathrm{K})$ and indirect $(\mathrm{Q}-\mathrm{K})$ excitonic states with energies of $E_{\mathrm{X}, \mathrm{D}}$ and $E_{\mathrm{X}, \mathrm{l}}$, respectively

trend $^{11,15-18}$. As the indirect gap energy is pushed away from or brought closer to the direct gap by strain, the exciton population participating in the direct-gap recombination will be affected by the presence of nearly degenerated indirect gap, leading to a modulation in the energy and intensity of direct exciton PL.

In this work, instead of applying external stress, we investigate the spatially resolved PL from monolayer $\mathrm{WSe}_{2}-\mathrm{MoS}_{2}$ and $\mathrm{MoSe}_{2}-\mathrm{WSe}_{2}$ lateral heterojunctions (HJs) with inherent nonuniform strain distribution ${ }^{19}$. The built-in local strain inhomogeneity exhibited in lateral $\mathrm{HJs}$ provides a unique platform to study the strain effects on $\mathrm{MX}_{2}$ without the need of applying strain. Here we first demonstrate PL from strained $\mathrm{MoS}_{2}$ in $\mathrm{WSe}_{2}-\mathrm{MoS}_{2}$ lateral HJs as a model system to extract the energy difference between the direct and indirect gaps in monolayer $\mathrm{MoS}_{2}$. Then we demonstrate the study of $\mathrm{MoSe}_{2}-\mathrm{WSe} \mathrm{e}_{2}$ lateral HJs and show that unstrained monolayer $\mathrm{WSe}_{2}$ is actually an indirect gap material.

\section{Results}

$\mathrm{PL}$ inhomogeneity in strained $\mathrm{WSe}_{2}-\mathrm{MoS}_{2}$ lateral HJs. Figure 1a shows the optical image of a typical $\mathrm{WSe}_{2}-\mathrm{MoS}_{2}$ lateral HJs. Raman analysis (Fig. 1b) reveals that the inner and outer regions are monolayer $\mathrm{WSe}_{2}$ and $\mathrm{MoS}_{2}$, respectively. The typical size of the HJ flakes is $\sim 15-25 \mu \mathrm{m}$. According to ref. ${ }^{19}$, the outer $\mathrm{MoS}_{2}$ exhibits nonuniform strain distribution due to the lattice mismatch between two materials, leading to considerable spatial variations in local PL spectra in the outer $\mathrm{MoS}_{2}$. Figure 1c shows five selected PL spectra, of which the PL energy variation is $100 \mathrm{meV}$, corresponding to a strain variation up to $\sim 2 \%$ in the monolayer $\mathrm{MoS}_{2}$ (based on the reported linear PL energy shift rate of $\sim 45 \mathrm{meV} / \%$ strain $)^{16}$. As shown in Fig. $1 \mathrm{~d}$ and $\mathrm{e}$, the spatial distributions of PL intensity and energy in outer $\mathrm{MoS}_{2}$ show a "positive" correlation, i.e., the site with a higher PL energy always exhibited a higher intensity. The PL intensity-energy correlation can be seen clearly in Fig. 1f, where the integrated 
intensities and peak energies collected from all local PL spectra of the outer $\mathrm{MoS}_{2}$ are plotted together.

The PL intensity-energy correlation arises from the variation of direct exciton populations caused by the strain-induced changes in the direct and indirect gap energies. For monolayer $\mathrm{MoS}_{2}$, the indirect gap $(\mathrm{K}-\Gamma)$ is higher in energy than the direct gap at K-valley (Fig. $1 \mathrm{~g})^{20-22}$. Since the $\mathrm{K}-\Gamma$ indirect gap $\left(E_{\mathrm{K} \Gamma}\right)$ reduces with tensile strain faster than the $\mathrm{K}-\mathrm{K}$ direct gap $\left(E_{\mathrm{KK}}\right)^{11,15-17}$, the direct and indirect exciton states will be brought closer in energy by tensile strain, leading to a marked change in direct exciton population and hence the PL intensity. We analyze the steady-state PL intensity by modeling the exciton populations in a simple two-level system (Supplementary Fig. 1 and Supplementary Note 1). In thermal equilibrium, the populations of direct $N_{\mathrm{X}, \mathrm{D}}$ and indirect $N_{\mathrm{X}, \mathrm{I}}$ excitons follow the Boltzmann distribution $N_{\mathrm{X}, \mathrm{I}}=N_{\mathrm{X}, \mathrm{D}} \exp \left(-\Delta / k_{\mathrm{B}} T\right)$, and

$$
N_{X, D}=\frac{N_{0}}{\exp \left(-\Delta / k_{\mathrm{B}} T\right)+1},
$$

where $\Delta=E_{\mathrm{X}, \mathrm{I}}-E_{\mathrm{X}, \mathrm{D}}$ is the energy difference between the indirect $\left(E_{\mathrm{X}, \mathrm{I}}\right)$ and direct $\left(E_{\mathrm{X}, \mathrm{D}}\right)$ exciton energies (Fig. 1h), and $N_{0}=N_{\mathrm{X}, \mathrm{D}}+N_{\mathrm{X}, \mathrm{I}}$ is the total exciton population. The energy difference $\Delta$ is strain dependent, which can be expressed as $\quad \Delta=\left(E_{\mathrm{X}, \mathrm{I}}^{0}+\delta E_{\mathrm{X}, \mathrm{I}}\right)-\left(E_{\mathrm{X}, \mathrm{D}}^{0}+\delta E_{\mathrm{X}, \mathrm{D}}\right)=\Delta_{0}+(\gamma-1) \delta E_{\mathrm{X}, \mathrm{D}}$, where $E_{\mathrm{X}, \mathrm{D}}^{0}$ and $E_{\mathrm{X}, \mathrm{I}}^{0}\left(\delta E_{\mathrm{X}, \mathrm{D}}\right.$ and $\left.\delta E_{\mathrm{X}, \mathrm{I}}\right)$ are the unstrained (strain-induced changes in) energies of the direct and indirect excitons, respectively, $\Delta_{0}=E_{\mathrm{X}, \mathrm{I}}^{0}-E_{\mathrm{X}, \mathrm{D}}^{0}$ is the unstrained value of $\Delta$, and $\gamma=\delta E_{\mathrm{X}, \mathrm{I}} / \delta E_{\mathrm{X}, \mathrm{D}}$, which is related to the ratio of deformation potential of the $\mathrm{K}-\Gamma$ and $\mathrm{K}-\mathrm{K}$ quasiparticle gaps. The strain-induced shift in the direct exciton energy, i.e., $\delta E_{\mathrm{X}, \mathrm{D}}=E_{\mathrm{X}, \mathrm{D}}-E_{\mathrm{X}, \mathrm{D}}^{0}$, can be obtained from the difference between PL peak energies of strained and unstrained monolayer $\mathrm{MoS}_{2}$. In our analysis, we assumed the PL peak energy of transferred $\mathrm{MoS}_{2}$ monolayers on sapphire substrates $(1.881 \mathrm{eV}$; vertical line in Fig. 1f) as the direct exciton energy of unstrained monolayer $\mathrm{MoS}_{2}$ (Supplementary Fig. 2). By fitting Eq. (1) to the experimental data and considering $\Delta_{0}$ and $\gamma$ as fitting parameters, we obtain a very good fit (solid curve in Fig. 1f) and determine $\Delta_{0}=0.08 \mathrm{eV}$ and $\gamma=1.95 \pm 0.14$ (see Supplementary Figs. 3 and 4 ), indicative of a direct optical gap in unstrained $\mathrm{MoS}_{2}$ monolayer, consistent with past understandings $7,8,20-22$.

$\mathrm{PL}$ inhomogeneity in strained $\mathrm{MoSe}_{2}-\mathrm{WSe}_{2}$ lateral $\mathrm{HJs}$. We now turn to the study of strained $\mathrm{WSe}_{2}$. Figure $2 \mathrm{a}$ shows the optical image of a typical $\mathrm{MoSe}_{2}-\mathrm{WSe}_{2}$ lateral $\mathrm{HJ}$, where the inner and outer regions have been identified as $\mathrm{MoSe}_{2}$ and $\mathrm{WSe}_{2}$ monolayers, respectively, by PL measurements (Fig. 2b). As shown in the spatially resolved PL measurements (Fig. 2c, d) and the selected PL spectra from different areas (Fig. 2e), the outer $\mathrm{WSe}_{2}$ region also exhibits remarkable variations in local PL intensity and energy due to the spatial strain inhomogeneity. Since the lattice mismatch between $\mathrm{MoSe}_{2}$ and $\mathrm{WSe}_{2}$ is small $(<0.2 \%)^{23}$, the strain inhomogeneity is likely to arise from the cooling process after high-temperature growth owing to the mismatch in thermal expansion coefficients between TMDs and substrates ${ }^{24-27}$. In contrary to strained $\mathrm{MoS}_{2}$, the PL intensity and energy of the strained $\mathrm{WSe}_{2}$ shows a "negative" correlation, i.e., the site with a higher PL energy exhibited a lower PL intensity (Fig. 2f). The negative intensity-energy correlation suggests that the strain-induced change in the indirect gap is very different from that in $\mathrm{MoS}_{2}$. In monolayer WSe 2 (Fig. 2g), the Q-K indirect gap is very close to $\mathrm{K}-\mathrm{K}$ direct gap. Under tensile strain, the $\mathrm{K}-\mathrm{K}$ direct gap reduces, while the $\mathrm{Q}-\mathrm{K}$ indirect gap increases ${ }^{18}$, leading to the increasing PL intensity with tensile strain.
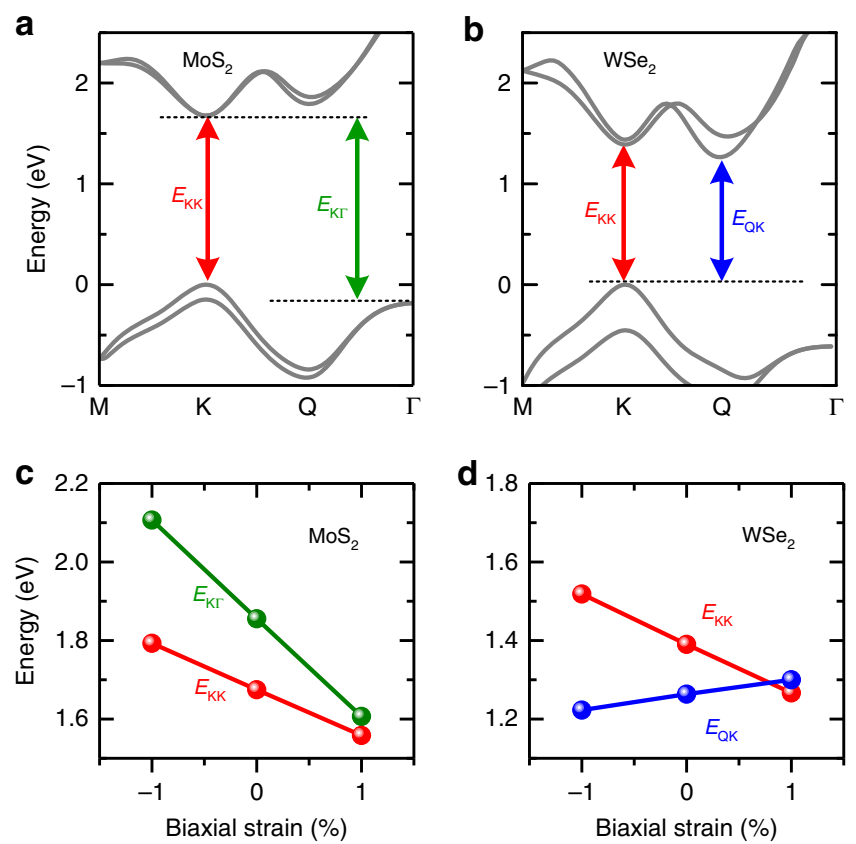

Fig. 3 DFT calculations of strained $\mathrm{MoS}_{2}$ and WSe $\mathrm{W}_{2}$ monolayers. a, b The calculated band structures for monolayer $\mathrm{MoS}_{2}(\mathbf{a})$ and $\mathrm{WSe}_{2}(\mathbf{b})$ based on DFT. $E_{\mathrm{KK}}$ and $E_{\mathrm{K} \Gamma}\left(E_{\mathrm{QK}}\right)$ denote the direct and the indirect gaps in $\mathrm{MoS}_{2}$ $\left(\mathrm{WSe}_{2}\right)$, respectively. c, d Strain-induced energy shifts in the direct and indirect gaps in monolayer $\mathrm{MoS}_{2}$ (c) and $\mathrm{WSe}_{2}$ (d). The calculations show that unstrained $\mathrm{WSe}_{2}$ is an indirect gap material, which can be turned into a direct-gap material by applying tensile strain

We use the same model to analyze the PL intensity-energy correlation of strained $\mathrm{WSe}_{2}$, but now the indirect exciton represent that formed at the Q-K indirect gap (Fig. $2 \mathrm{~h}$ ). We have also determined $E_{\mathrm{X} . \mathrm{D}}^{0}=1.661 \mathrm{eV}$ for unstrained monolayer $\mathrm{WSe}_{2}$ (vertical line in Fig. 2f) according to PL measurements on transferred $\mathrm{WSe}_{2}$ monolayers (Supplementary Fig. 2). As shown by the solid curve in Fig. $2 \mathrm{f}$, the model fits the data very well, yielding $\Delta_{0}=-0.07 \mathrm{eV}$ and $\gamma=-0.32 \pm 0.05$ (Supplementary Fig. 5). Interestingly, both $\Delta_{0}$ and $\gamma$ for monolayer $\mathrm{WSe}_{2}$ are negative, in stark contrast to monolayer $\mathrm{MoS}_{2}$. The negative $\gamma$ arises from the opposite energy evolutions of $\mathrm{Q}$ and $\mathrm{K}$ valleys with strain ${ }^{18}$, while the negative $\Delta_{0}$ is a direct evidence of indirect optical gap in unstrained monolayer $\mathrm{WSe}_{2}$. The indirect gap of monolayer $\mathrm{WSe}_{2}$ also manifested itself in the large variation in PL intensity (up to a factor of $\sim 5$ ) shown in Fig. $2 \mathrm{f}$, because, according to Eq. (1), the increase in PL intensity by tensile strain should be less than a factor of two if monolayer $\mathrm{WSe}_{2}$ is initially a direct gap material.

Calculated strain-induced bandgap shifts in $\mathrm{MoS}_{2}$ and WSe monolayers. Band structure calculations based on density functional theory (DFT) have been performed and compared to our experimental results. As shown in Fig. 3a, the calculated band structure for unstrained monolayer $\mathrm{MoS}_{2}$ exhibits a direct gap at the $\mathrm{K}$ valleys. The calculated energies of the $\mathrm{K}-\mathrm{K}$ and $\mathrm{K}-\Gamma$ gaps as a function of strain (Fig. 3c) show a faster decrease in $E_{\mathrm{K} \Gamma}$ with tensile strain than $E_{\mathrm{KK}}$. If we ignore the strain-induced change in exciton binding energy, the ratio of strain-induced changes in the $\mathrm{K}-\Gamma$ and $\mathrm{K}-\mathrm{K}$ gaps (i.e., $\delta E_{\mathrm{K} \Gamma} / \delta E_{\mathrm{KK}}$ ) thus represents the ratio of strain-induced changes in the optical gaps $\gamma=\delta E_{\mathrm{X}, \mathrm{I}} / \delta E_{\mathrm{X}, \mathrm{D}}$. From the calculations, we determine $\delta E_{\mathrm{K} \Gamma} / \delta E_{\mathrm{KK}}=2.13$, consistent with our experimentally obtained $\gamma=1.95 \pm 0.14$ for monolayer $\mathrm{MoS}_{2}$. On the contrary, our calculations show that unstrained monolayer $\mathrm{WSe}_{2}$ exhibits an indirect gap between the 
a

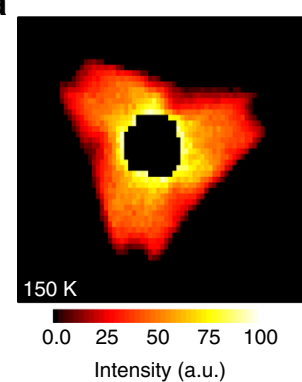

C

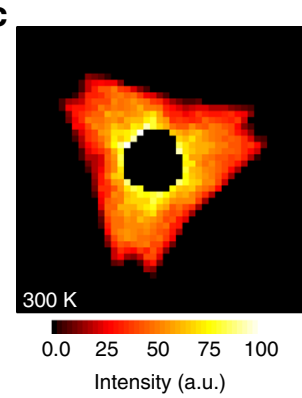

b

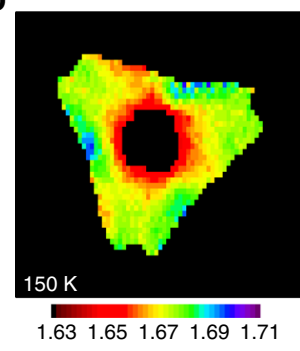

Energy (eV)

d

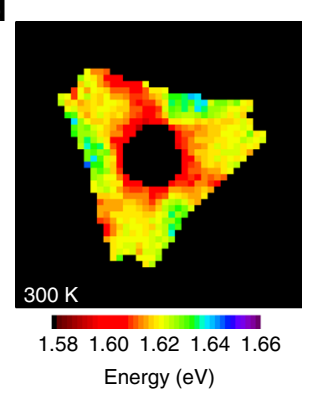

e

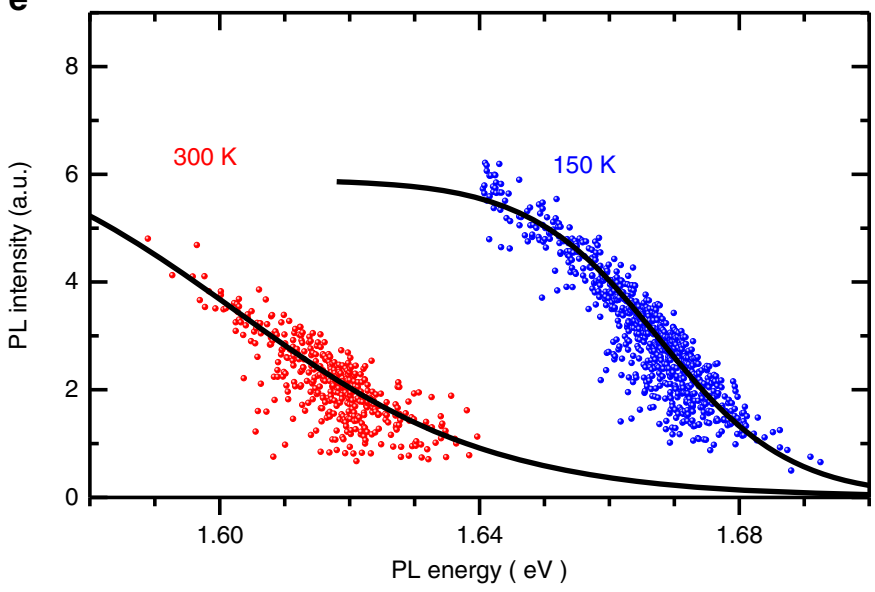

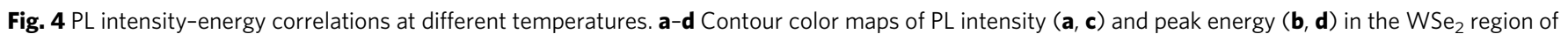

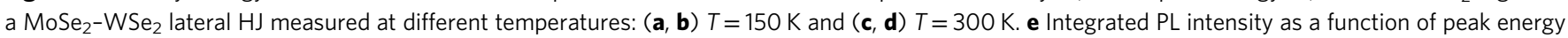
measured at $150 \mathrm{~K}$ (blue dots) and $300 \mathrm{~K}$ (red dots). Black lines are model fitting curves

Q-valley in the $\mathrm{CB}$ and the $\mathrm{K}$ point in the valence band (VB), as shown in Fig. 3b. Applying tensile strain (Fig. 3d) tends to shrink the $\mathrm{K}-\mathrm{K}$ gap, but expand the $\mathrm{Q}-\mathrm{K}$ gap. We determine $\delta E_{\mathrm{QK}} / \delta E_{\mathrm{KK}}=-0.28$, in good agreement with our experimental value of $\gamma=-0.32 \pm 0.05$ for monolayer $\mathrm{WSe}_{2}$.

We emphasize that the experimentally determined $\Delta_{0}$ does not reflect the actual energy difference between the direct and indirect quasiparticle gaps. Due to the strong exciton binding energy caused by reduced dielectric screening in monolayer TMDs $6,28,29$, the difference in binding energies of direct and indirect excitons has to be taken into account. We first calculate the direct exciton binding energy $E_{\mathrm{B}, \mathrm{D}}$ based on the effective 2D Coulomb potential (Supplementary Fig. 6 and Supplementary Note 2). The indirect exciton binding energy $E_{\mathrm{B}, \mathrm{I}}$ is then estimated by scaling with the effective reduced masses deduced from band structure calculations. We obtain $E_{\mathrm{B}, \mathrm{D}}=0.23 \mathrm{eV}(\mathrm{K}-\mathrm{K})$ and $E_{\mathrm{B}, \mathrm{I}}=0.26 \mathrm{eV}(\mathrm{K}-\Gamma)$ for monolayer $\mathrm{MoS}_{2}$. The higher $E_{\mathrm{B}, \mathrm{I}}$ by $0.03 \mathrm{eV}$, originating from the heavier hole effective mass at $\Gamma$ valley, implies that the experimentally determined $\Delta_{\mathrm{eV}}=0.08 \mathrm{eV}$ underestimates the actual energy difference between the $\mathrm{K}$ - and $\Gamma$-valleys in the $\mathrm{VB}$, yielding $\Delta_{\mathrm{K}-\Gamma}=E_{\mathrm{K} \Gamma}-E_{\mathrm{KK}} \approx 0.11 \mathrm{eV}$, in good agreement with the value in the range of $0.13-0.30 \mathrm{eV}$ determined by angle-resolved photoemission spectroscopy ${ }^{20-22}$. For monolayer $\mathrm{WSe}_{2}$, due to the similar effective mass of electrons at $\mathrm{K}$ - and Q-valleys in the $\mathrm{CB}$, the deduced $E_{\mathrm{B}, \mathrm{D}}=0.19 \mathrm{eV}(\mathrm{K}-\mathrm{K})$ and $E_{\mathrm{B}, \mathrm{I}}=0.20 \mathrm{eV}(\mathrm{Q}-\mathrm{K})$ are also similar, yielding $\Delta_{\mathrm{K}-\mathrm{Q}}=$ $E_{\mathrm{QK}}-E_{\mathrm{KK}} \approx-0.06 \mathrm{eV}$. It demonstrates that monolayer $\mathrm{WSe}_{2}$ exhibits not only an indirect optical gap, but also an indirect quasiparticle gap with a $\mathrm{CB}$ minimum at the Q-valley, which is $\sim 60 \mathrm{meV}$ lower than the K-valley, consistent with STS measurements ${ }^{14}$.

Temperature dependence of PL intensity-energy correlation. We noted that the direct exciton populations in the simple two-level model depend sensitively on temperature. To attest the validity of our model analysis, we have performed the same measurements of spatial PL mapping on another $\mathrm{MoSe}_{2}-\mathrm{WSe}_{2} \mathrm{HJ}$ flake at $\mathrm{T}=150$ and $300 \mathrm{~K}$, as shown in Fig. $4 \mathrm{a}-\mathrm{d}$. The corresponding PL intensity-energy correlations of the outer monolayer $\mathrm{WSe}_{2}$ at 150 and $300 \mathrm{~K}$ are shown in Fig. 4e. Apart from a rigid shift in PL energy, the PL intensity measured at $\mathrm{T}=150 \mathrm{~K}$ exhibits a sharper change with energy due to the reduced thermal energy $k_{\mathrm{B}} T$ at a lower temperature. The simple two-level model can fit the two data sets with the same $\Delta_{0}$ and $\gamma$-values (solid curves in Fig. 4e) by simply changing the sample temperature and the unstrained PL peak energy. The temperature dependent measurements thus further confirm that the observed PL intensity-energy correlation is governed by the interplay of direct and indirect exciton populations. The nonuniform distribution of nonradiative defects or inhomogeneous alloy mixing can thus be excluded from the origin of the observed PL intensity-energy correlation.

\section{Discussion}

In our analysis, we neglect the influence of dark exciton states at $\mathrm{K}$ valleys arising from the spin splitting in the $\mathrm{CB}$. In particular, the spin-forbidden $\mathrm{K}-\mathrm{K}$ dark exciton state is lower in energy by $\sim 30 \mathrm{meV}$ in monolayer $\mathrm{WSe}_{2}{ }^{30}$, which also affects the bright (direct) exciton populations and hence the PL intensity at different temperatures. However, because the strain-induced change in the $\mathrm{CB}$ spin splitting is very small $(<0.5 \mathrm{meV} / \%)^{31}$, including the dark exciton states will not change the analysis of $\Delta_{0}$ and $\gamma$-values from the PL intensity-energy correlation measured at a given temperature.

Strain could also impact the radiative lifetime and the binding energy of the $\mathrm{K}-\mathrm{K}$ direct exciton through modifications in wave functions, optical matrix element and band curvatures. However, according to the range of PL shifts in our samples and recent theoretical calculations ${ }^{32}$, we estimated that the strain-induced changes in the radiative lifetime and the binding energy are small $(<5 \%)$, which is within our experimental uncertainty.

Spatial variation of the unintentional doping in the sample could also lead to PL shifts due to the charged exciton (trion) emissions. We have performed line-shape analysis for the selected PL spectra shown in Fig. 2e and found that no spectral signature can be attributed to the trion emissions (Supplementary Fig. 7). 
Therefore, spatial variation in doping level can be excluded from the observed PL shift and the intensity-energy correlation.

Alloy inhomogeneity caused by material intermixing could be another source of the observed PL inhomogeneity. We have measured the PL inhomogeneity for $\mathrm{HJ}$ flakes after ultrasonic treatments. Interestingly, we found that the spatial distributions of PL intensity and energy are changed, but still exhibit a similar negative correlation (Supplementary Fig. 8). Since the strain inhomogeneity is likely to form during the fast-cooling process caused by the contraction mismatch, the local interactions between TMD and the substrate could be loosen and redistributed by ultrasonic treatments, and thereby changing the pattern of strain inhomogeneity. This result thus excludes the possibility that the PL inhomogeneity is caused by local variation of doping, defect distribution or alloy composition, since they are unlikely to be altered by ultrasonic treatments.

The indirect optical gap in unstrained monolayer $\mathrm{WSe}_{2}$ identified in this work has important implications. As a light emitter, monolayer $\mathrm{WSe}_{2}$ is far from ideal as compared with other $\mathrm{MX}_{2}$ with a direct gap, since a majority of photogenerated carriers will form indirect excitons even at room temperature, which considerably limits the quantum yield of light emissions. On the other hand, the efficient light absorption through the direct optical gap, together with the suppressed radiative recombination efficiency for the indirect excitons, makes monolayer $\mathrm{WSe}_{2}$ very ideal for photovoltaic applications. The indirect Q-K exciton in $\mathrm{WSe}_{2}$ is essentially dark and inaccessible optically. Recent theoretical work predicted that non-covalently attached molecules with a strong dipole moment can turn the indirect dark exciton bright, implicating its applications in molecule sensing ${ }^{33}$. Nevertheless, applying a moderate tensile strain $(\sim 1 \%$ uniaxial or $\sim 0.5 \%$ biaxial) can tune the bandgap from indirect to direct, which can improve drastically the emission efficiency by nearly an order of magnitude. Based on the simple two-level model, we estimated that the crossover of the direct and indirect optical gaps occurs at $1.607 \mathrm{eV}(1.797 \mathrm{eV})$ for monolayer $\mathrm{WSe}_{2}\left(\mathrm{MoS}_{2}\right)$, as indicated by the vertical dash line in Fig. 2f (Fig. 1f). For the strained $\mathrm{MoSe}_{2}-\mathrm{WSe}_{2}$ lateral HJs investigated here, the PL peak energy of the $\mathrm{WSe}_{2}$ region spans from 1.58 to $1.64 \mathrm{eV}$, which covers the crossover energy of direct and direct optical gap at $1.607 \mathrm{eV}$. This means that local strain variation also gives rise to a spatial modulation of direct and indirect gap, which forms a very unique platform with nonuniform potential modulations for studying exciton diffusions and localizations. Finally, we point out that the indirect gap nature of monolayer $\mathrm{WSe}_{2}$ must to be taken into account in further studies of its fundamental properties (e.g., exciton spin valley dynamics) and device applications for light emissions and harvesting.

\section{Methods}

Growth of monolayer lateral heterojunctions. High-quality single-crystal TMD HJs were synthesized on sapphire substrates by chemical vapor deposition (CVD) in horizontal hot-wall chambers. The monolayer $\mathrm{WSe}_{2}-\mathrm{MoS}_{2}$ lateral HJs were grown using the two-step growth method (see ref. ${ }^{19}$ for details), while $\mathrm{MoSe}_{2}-\mathrm{WSe}_{2}$ lateral HJs were grown using the conventional one-pot synthesis process. For $\mathrm{WSe}_{2}-\mathrm{MoS}_{2} \mathrm{HJs}$, we used high-purity $\mathrm{WO}_{3}, \mathrm{MoO}_{3}$, Se and S powders as the source precursors. The growth temperatures for $\mathrm{WSe}_{2}$ and $\mathrm{MoS}_{2}$ are 925 and $755^{\circ} \mathrm{C}$, respectively. For $\mathrm{MoSe}_{2}-\mathrm{WSe}_{2} \mathrm{HJs}$, high-purity $\mathrm{MoO}_{2}, \mathrm{WO}_{3}$, and Se powder were used as the initial reactants, and the $\mathrm{HJs}$ were grown at $880^{\circ} \mathrm{C}$. All growths were performed in $\mathrm{Ar} / \mathrm{H}_{2}$ flowing gas at low pressure (5-40 Torr).

Optical measurements. PL and Raman measurements were performed using a home-built optical microscope in the back-scattering configuration. A $532 \mathrm{~nm}$ solid-state laser was used as the excitation source. The laser were focused on the sample by a $100 \times$ objective lens $(\mathrm{NA}=0.9)$. The PL and Raman signals were collected by the same objective, analyzed by a $0.75-\mathrm{m}$ monochromator and detected by a nitrogen-cooled CCD camera. For confocal measurements, the sample image was projected onto a $50 \mu \mathrm{m}$ pinhole before entering the monochromator, yielding an overall spatial resolution of $\sim 0.5 \mu \mathrm{m}$. Room temperature spatial mappings of PL spectra were performed using a fast motorized $x-y$ stage with a step size of $0.25 \mu \mathrm{m}$. Low-temperature measurements were performed in a cryogen-free and vibrationfree cryostat equipped with a 3-axis piezo-positioner, an $x-y$ scanner and an objective lens (N.A. $=0.82$ ) in the low-temperature chamber.

DFT calculations. The first-principle calculations were based on the generalized gradient approximation $(\mathrm{GGA})^{34}$ using the full-potential projected augmented wave method ${ }^{35,36}$ as implemented in the Vienna ab initio simulation (VASP) package ${ }^{37,38}$. The electronic structures of monolayer $\mathrm{MoS}_{2}$ and $\mathrm{WSe}_{2}$ were calculated using a $30 \times 30 \times 1$ Monkhorst-Pack $k$-mesh over the Brillouin zone with a cutoff energy of $500 \mathrm{eV}$ under geometry optimization. The spin-orbit coupling was included in a self-consistent manner. Slabs with vacuum thickness larger than $30 \AA$ were used to model the thin films. For the strain effects, the atomic positions were relaxed until the residual forces were less than $0.001 \mathrm{eV} / \AA$.

Data availability. The data that support the findings of this study are available from the corresponding author upon reasonable request.

Received: 11 May 2017 Accepted: 11 August 2017

Published online: 13 October 2017

\section{References}

1. Wang, Q. H., Kalantar-Zadeh, K., Kis, A., Coleman, J. N. \& Strano, M. S. Electronics and optoelectronics of two-dimensional transition metal dichalcogenides. Nat. Nanotechnol. 7, 699-712 (2012).

2. Jariwala, D., Sangwan, V. K., Lauhon, L. J., Marks, T. J. \& Hersam, M. C. Emerging device applications for semiconducting two-dimensional transition metal dichalcogenides. ACS Nano 8, 1102-1120 (2014).

3. Fiori, G. et al. Electronics based on two-dimensional materials. Nat. Nanotechnol. 9, 768-779 (2014).

4. Akinwande, D., Petrone, N. \& Hone, J. Two-dimensional flexible nanoelectronics. Nat. Commun. 5, 5678 (2014).

5. Xia, F., Wang, H., Xiao, D., Dubey, M. \& Ramasubramaniam, A. Twodimensional material nanophotonics. Nat. Photon 8, 899-907 (2014).

6. Mak, K. F. \& Shan, J. Photonics and optoelectronics of 2D semiconductor transition metal dichalcogenides. Nat. Photon 10, 216-226 (2016).

7. Splendiani, A. et al. Emerging photoluminescence in monolayer $\mathrm{MoS}_{2}$. Nano Lett. 10, 1271-1275 (2010)

8. Mak, K. F., Lee, C., Hone, J., Shan, J. \& Heinz, T. F. Atomically thin $\mathrm{MoS}_{2}$ : A new direct-gap semiconductor. Phys. Rev. Lett. 105, 136805 (2010).

9. Ding, Y. et al. First principles study of structural, vibrational and electronic properties of graphene-like $\mathrm{MX}_{2}(\mathrm{M}=\mathrm{Mo}, \mathrm{Nb}, \mathrm{W}, \mathrm{Ta} ; \mathrm{X}=\mathrm{S}, \mathrm{Se}, \mathrm{Te})$ monolayers. Phys. B 406, 2254-2260 (2011).

10. Zhu, Z. Y., Cheng, Y. C. \& Schwingenschlögl, U. Giant spin-orbit-induced spin splitting in two-dimensional transition-metal dichalcogenide semiconductors. Phys. Rev. B 84, 153402 (2011).

11. Yun, W. S., Han, S. W., Hong, S. C., Kim, I. G. \& Lee, J. D. Thickness and strain effects on electronic structures of transition metal dichalcogenides: $2 \mathrm{H}-M \mathrm{X}_{2}$ semiconductors ( $M=\mathrm{Mo}, \mathrm{W} ; X=\mathrm{S}, \mathrm{Se}, \mathrm{Te})$. Phys. Rev. B 85, 033305 (2012).

12. Liang, Y., Huang, S., Soklaski, R. \& Yang, L. Quasiparticle band-edge energy and band offsets of monolayer of molybdenum and tungsten chalcogenides. Appl. Phys. Lett. 103, 042106 (2013).

13. Ramasubramaniam, A. Large excitonic effects in monolayers of molybdenum and tungsten dichalcogenides. Phys. Rev. B 86, 115409 (2012).

14. Zhang, C. et al. Probing critical point energies of transition metal dichalcogenides: Surprising indirect gap of single layer $\mathrm{WSe}_{2}$. Nano Lett. 15, 6494-6500 (2015).

15. He, K., Poole, C., Mak, K. F. \& Shan, J. Experimental demonstration of continuous electronic structure tuning via strain in atomically thin $\mathrm{MoS}_{2}$. Nano Lett. 13, 2931-2936 (2013).

16. Conley, H. J. et al. Bandgap engineering of strained monolayer and bilayer $\mathrm{MoS}_{2}$. Nano Lett. 13, 3626-3630 (2013).

17. Zhu, C. R. et al. Strain tuning of optical emission energy and polarization in monolayer and bilayer $\mathrm{MoS}_{2}$. Phys. Rev. B 88, 121301(R) (2013).

18. Desai, S. B. et al. Strain-induced indirect to direct bandgap transition in multilayer $\mathrm{WSe}_{2}$. Nano Lett. 14, 4592-4597 (2014).

19. Li, M.-Y. et al. Epitaxial growth of a monolayer $\mathrm{WSe}_{2}-\mathrm{MoS}_{2}$ lateral p-n junction with an atomically sharp interface. Science 349, 524-528 (2015).

20. Jin, W. et al. Direct measurement of the thickness-dependent electronic band structure of $\mathrm{MoS}_{2}$ using angle-resolved photoemission spectroscopy. Phys. Rev. Lett. 111, 106801 (2013).

21. Chiu, M.-H. et al. Determination of band alignment in the single-layer $\mathrm{MoS}_{2} / \mathrm{WSe}_{2}$ heterojunction. Nat. Commun. 6, 7666 (2015).

22. Yuan, H. et al. Evolution of the valley position in bulk transition-metal chalcogenides and their monolayer limit. Nano Lett. 16, 4738-4745 (2016). 
23. Brixner, L. H. Preparation and properties of the single crystalline $\mathrm{AB}_{2}$-type selenides and tellurides of niobium, tantalum, molybdenum and tungsten. J. Inorg. Nucl. Chem 24, 257-263 (1962).

24. Amani, M. et al. Growth-substrate induced performance degradation in chemically synthesized monolayer $\mathrm{MoS}_{2}$ field effect transistors. Appl. Phys. Lett. 104, 203506 (2014).

25. Liu, Z. et al. Strain and structure heterogeneity in $\mathrm{MoS}_{2}$ atomic layers grown by chemical vapour deposition. Nat. Commun. 5, 5246 (2014).

26. Park, K.-D. et al. Hybrid tip-enhanced nanospectroscopy and nanoimaging of monolayer $\mathrm{WSe}_{2}$ with local strain control. Nano Lett. 16, 2621-2627 (2016).

27. Amani, M. et al. High luminescence efficiency in $\mathrm{MoS}_{2}$ grown by chemical vapor deposition. ACS Nano 10, 6535-6541 (2016).

28. Zhang, C., Johnson, A., Hsu, C.-L., Li, L.-J. \& Shih, C.-K. Direct imaging of band profile in single layer $\mathrm{MoS}_{2}$ on graphite: Quasiparticle energy gap, metallic edge states, and edge band bending. Nano Lett. 14, 2443-2447 (2014).

29. He, K. et al. Tightly bound excitons in monolayer WSe $\mathrm{W}_{2}$. Phys. Rev. Lett. 113 , 026803 (2014)

30. Zhang, X.-X., You, Y., Zhao, S. Y. F. \& Heinz, T. F. Experimental evidence for dark excitons in monolayer WSe 2 . Phys. Rev. Lett. 115, 257403 (2015).

31. Cheiwchanchamnangij, T. \& Lambrecht, W. R. L. Strain effects on the spinorbit-induced band structure splittings in monolayer $\mathrm{MoS}_{2}$ and graphene. Phys. Rev. B 88, 155404 (2013).

32. Feierabend, M., Morlet, A., Berghäuser, G. \& Malic, E. Impact of strain on the optical fingerprint of monolayer transition metal dichalcogenides. Phys. Rev. B 96, 045245 (2017)

33. Feierabend, M., Berghäuser, G., Knorr, A. \& Malic, E. Proposal for dark exciton based chemical sensors. Nat. Commun. 8, 14776 (2017).

34. Perdew, J. P., Burke, K. \& Ernzerhof, M. Generalized gradient approximation made simple. Phys. Rev. Lett. 77, 3865-3868 (1996).

35. Blöchl, P. E. Projector augmented-wave method. Phys. Rev. B 50, 17953-17979 (1994).

36. Kresse, G. \& Joubert, D. From ultrasoft pseudopotentials to the projector augmented-wave method. Phys. Rev. B 59, 1758-1775 (1999).

37. Kresse, G. \& Hafner, J. Ab initio molecular dynamics for open-shell transition metals. Phys. Rev. B 48, 13115-13118 (1993).

38. Kresse, G. \& Furthmüller, J. Efficiency of $a b$-initio total energy calculations for metals and semiconductors using a plane-wave basis set. Comput. Mater. Sci. 6, 15-50 (1996).

\section{Acknowledgements}

This work was supported by the Ministry of Science and Technology (MOST) of Taiwan (MOST-104-2628-M-009-002-MY3, MOST-105-2119-M-009-014-MY3) and AOARD
(FA2386-16-1-4035). W.-H.C. acknowledges the supports from the Center for Interdisciplinary Science of NCTU. T.-R.C. acknowledges the supports from MOST and NCKU. H.-T.J. acknowledges the supports from MOST, NTHU and Academia Sinica. T.-R.C. and H.-T.J. also thank NCHC, CINC-NTU and NCTS for technical supports.

\section{Author contributions}

W.-H.C. and W.-T.H. conceived the idea and designed the experiment. W.-T.H and D. W. performed the spectroscopy measurements. The samples were grown and characterized by L.-S.L, J.-K.H and M.-Y.L., and assisted by Z.-Y.J., Y.-C.C and L.-J.L. T.-R.C. and H.-T.J. performed DFT calculations. W.-H.C. and W.-T.H. wrote the paper. All authors discussed the results and commented on the manuscript.

\section{Additional information}

Supplementary Information accompanies this paper at doi:10.1038/s41467-017-01012-6.

Competing interests: The authors declare no competing financial interests.

Reprints and permission information is available online at http://npg.nature.com/ reprintsandpermissions/

Publisher's note: Springer Nature remains neutral with regard to jurisdictional claims in published maps and institutional affiliations.

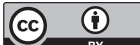

Open Access This article is licensed under a Creative Commons Attribution 4.0 International License, which permits use, sharing, adaptation, distribution and reproduction in any medium or format, as long as you give appropriate credit to the original author(s) and the source, provide a link to the Creative Commons license, and indicate if changes were made. The images or other third party material in this article are included in the article's Creative Commons license, unless indicated otherwise in a credit line to the material. If material is not included in the article's Creative Commons license and your intended use is not permitted by statutory regulation or exceeds the permitted use, you will need to obtain permission directly from the copyright holder. To view a copy of this license, visit http://creativecommons.org/ licenses/by/4.0/

(C) The Author(s) 2017 